\title{
Properties and Protein-resistance of Self-assembled Film by Amphiphilic Fluoropolymers
}

\author{
Hong GUO and Ling $\mathrm{HE}^{*}$
}

Department of Chemistry, School of Science, Xi'an Jiaotong University, Xianning West Road, 28, Xi'an

\author{
710049, China \\ * heling@mail.xjtu.edu.cn \\ *Corresponding author *Tel: 0086-29-82668554, *Fax: 0086-29-82668559
}

Keywords: Amphiphilic fluoropolymer, Self-assembly colloids, Solvent-responsive, Surface properties, Thermal stability, Protein-resistance, Coatings.

\begin{abstract}
Amphiphilic fluorocopolymers P(MMA)-co-P(PEGMA)-co-P(DFHM) are prepared via ATRP by hydrophilic poly(ethylene glycol) methyl ether methacrylate (PEGMA), hydrophobic dodecafluorohrptyl methacrylate (DFHM) and methyl methacrylate (MMA). The self-assembled surface is characterized by chemical composition, morphology, wettability and the protein adsorption resistance to bovine serum albumin (BSA). The hemispherical micelles are composed of light semisphere $\mathrm{P}(\mathrm{MMA})$-co-P(PEGMA) and dark semisphere $\mathrm{P}(\mathrm{DFHM})$ in $\mathrm{CHCl}_{3}$. With the diameters of P(MMA)-co-P(PEGMA)-co-P(DFHM) micelles increased by the decreasing of fluorine content in MMA/PEGMA/DFHM, as 180nm (14/1/1.2), 230nm (20/1/0.8) and 280nm (14/1/0.4), the surface roughness of films is accordingly reduced as $0.343 \mathrm{~nm}, 0.754 \mathrm{~nm}$ and $1.01 \mathrm{~nm}$. During the film-forming, the fluoride segments migrate onto the film surface to contain rounded-hills or cylindrical-shape distributed morphology. Much high hexadecane contact angle $\left(54.73^{\circ}-55.41^{\circ}\right)$ and water contact angle $\left(122.74^{\circ}\right)$ of film surfaces are able to indicate the sufficient hydrophilic/hydrophobic surface, but the film by much contain PEGMA content shows an excellent behavior of BSA protein-resistance $(\triangle \mathrm{f}=-15.0 \mathrm{~Hz}$ ). When the MMA/PEGMA/DFHM is controlled at 20/1/0.8 and 14/1/0.4, the amphiphilic fluorocopolymers obtain much high $\mathrm{Td}\left(203.4^{\circ} \mathrm{C}\right)$.
\end{abstract}

\section{Introduction}

Amphiphilic copolymer has provided wide applications in advanced materials, nanotechnology, special surface properties and medicine carrier by self-assembled different nanoparticles in selected solutions. Normally, the synthesis of amphiphilic copolymer is the traditional free radical polymerization [1], atom transfer radical polymerization, reversible addition-fragmentation chain transfer (RAFT) polymerization, stable free radical polymerization and condensation polymerization. Among of them, ATRP is the most widely used technique because of mild reaction conditions and simple experimental operation.

In the amphiphilic copolymers, the hydrophilic segment offered by poly (ethylene glycol) methyl ether methacrylate (PEGMA) contains a linear methacrylate backbone and reactive functional side chain of poly(ethylene glycol) (PEG) [2], therefore, it could produce a wealth of copolymer materials, such as temperature-sensitive poly-N-isopropyl acrylamide-co-polyethylene glycol methyl methacrylates (NPGs) [3] and poly (PEGMA-co-MMA-co-acryloyl- $\beta$-CD) that has a potential application on pharmaceutics [4]. On the other hand, the interaction between P(PEGMA) with water molecules or other solvents containing hydrogen by methoxy, ethoxy or hydroxy in the hydrophilic side chain [5] to make it as the materials used in the protein adsorption resistance because of its stereo-hindrance effect [6,7]. While, the hydrophobic segment provided by fluoropolymer could produce low surface tension and excellent chemical/physical properties [8-10], such as the 
alkyne-P(HEMA-co-MMA)-b-PPEGMA [11] and a brush-shaped block amphiphilic fluorinated copolymers P(PEGMA-co-MMA)-b-PC6SMA [12]. Furthermore, in order to improve the hardness of amphiphilic copolymers, poly (methyl methacrylate) (PMMA) is suggested to be introduced into copolymer with excellent physical/chemical properties [13]. Therefore, based on the excellent performance of random-structured amphiphilic copolymer in protein adsorption resistance, a random amphiphlic copolymer with hydrophobic fluoro-segment and PMMA, and hydrophilic P(PEGMA) segment is hoped to create a coating-film in protein adsorption resistance.

In this paper, the amphiphilic fluorocopolymers are synthesized by hydrophobic dodecafluorohrptyl methacrylate (DFHM), methyl methacrylate (MMA), and hydrophilic poly(ethylene glycol) methyl ether methacrylate (PEGMA) via ATRP technique. The self-assembly behavior and surface properties of P(PMMA)-co-P(PEGMA)-co-P(DFHM) film are characterized. The protein adsorption resistance of film surface is monitored by quartz crystal microbalance dissipation (QCM-D).

\section{Experimental studies}

Materials. Poly(ethylene glycol) methyl ether methacrylate (PEGMA, $\left.\left(\mathrm{C}_{2} \mathrm{H}_{4} \mathrm{O}\right) \mathrm{nC}_{5} \mathrm{H}_{8} \mathrm{O}_{2}\right)$ was purchased by Aladdin Industrial Corporation. Dodecafluoroheptyl methacrylate (DFHM, $\mathrm{C}_{11} \mathrm{H}_{8} \mathrm{O}_{2} \mathrm{~F}_{12}$, liquid) was supplied by XEOGIA Fluorine-Silicon Chemical Co. Ltd (China). Methylmethacrylate (MMA, 99wt\%, Aldrich), cuprous chloride $(\mathrm{CuCl})$, cyclohexanone and tetrahydrofuran (THF) was purified prior to use. N,N,N',N",N"-pentamethyldiethylenetriamine (PMDETA, $\mathrm{C}_{9} \mathrm{H}_{23} \mathrm{~N}_{3}$ ) was supplied by Energy Chemical Co. Ltd (China). Analytical purity of ethyl 2-bromoisobutyrate (EBiB), chloroform $\left(\mathrm{CHCl}_{3}\right)$, n-pentane were obtained commercially and used as-received.

Synthesis of amphiphilic fluorocopolymer P(MMA)-co-P(PEGMA)-co-P(DFHM). ATRP approach was used to synthesize P(MMA)-co-P(PEGMA)-co-P(DFHM), and the process was shown in Scheme 1. When a Schlenk flask was dried under an inert atmosphere of nitrogen, a mixture of EBiB initiator, MMA, PEGMA, DFHM, PMEDTA and cyclohexanone was introduced using degassed syringes after deoxygenation. $\mathrm{CuCl}$ was placed in an evacuated and flushed Schlenk flask. The molar ratio of [EBiB]:[CuCl]:[PMDETA] was controlled as $1: 1: 1$, and the molar ratio of [MMA]:[PEGMA]:[DFHM] was set to 14:1:1.2, 20:1:0.8 and 14:1:0.4 (Table.1). Then, the solution was vigorously stirred and heated to $10{ }^{\circ} \mathrm{C}$ in an oil-bath. After $24 \mathrm{~h}$ reaction, THF was added to dilute the reaction system. The solution was passed through a basic alumina column to remove the catalyst and the solvent under vacuum, followed by adding drop wisely the viscous liquid product into n-pentane. Finally, dumping out the upper n-pentane and drying the product in a vacuum oven at $45{ }^{\circ} \mathrm{C}$ overnight, The transparent solid of P(MMA)-co-P(PEGMA)-co-P(DFHM) copolymer was obtained.

The chemical structure of P(MMA)-Co-P(PEGMA)-co-P(DFHM) has been confirmed by ${ }^{1} \mathrm{H}-\mathrm{NMR}$ spectroscopy (Fig.1). The peaks at $0.84 \mathrm{ppm}$ (a)-2.04ppm (b) are ascribed to the backbone of $-\mathrm{CH}_{2}-$ and $-\mathrm{CH}_{3}$ in MMA, PEGMA and PDFHM, respectively. The superposition signal at 3.38ppm is $-\mathrm{OCH}_{3}$ group in MMA(c) and PEGMA(d). The peak at $4.2 \mathrm{ppm}$ (e) is assigned to $-\mathrm{CH}_{2}$ - in PEGMA side chains next to the $-\mathrm{OC}=\mathrm{O}$ group and the signals at 3.50-3.74 ppm (f) are contributed by methylene $\left(-\mathrm{OCH}_{2}-\mathrm{CH}_{2}-\right)$. The characteristic signals at $5.55 \mathrm{ppm}(\mathrm{g})$ and $4.45 \mathrm{ppm}$ are formed by -CFH and $-\mathrm{OCH}_{2}$ groups in PDFHM. Furthermore, the molecular weight of P(PEGMA)-Co-P(DFHM) achieved by GPC analysis is $2.902 \times 10^{4}, 2.302 \times 10^{4}$ and $2.102 \times 10^{4} \mathrm{~g} \cdot \mathrm{mol}^{-1}$ for three molar ratio of MMA/PEGMA/DFHM=14/1/1.2, 20/1/0.8 and 14/1/0.4 (Table 1). The relatively low polydispersity index (PDI=1.11-1.47) reveals a narrow distribution of molecular weight obtained by less DFHM content. The ${ }^{1} \mathrm{H}-\mathrm{NMR}$ and molecular weight results have proved that the random copolymers have been successfully obtained and proceed for the employed feed ratios, reactant concentrations and reaction conditions (Table.1 and Scheme.1). 


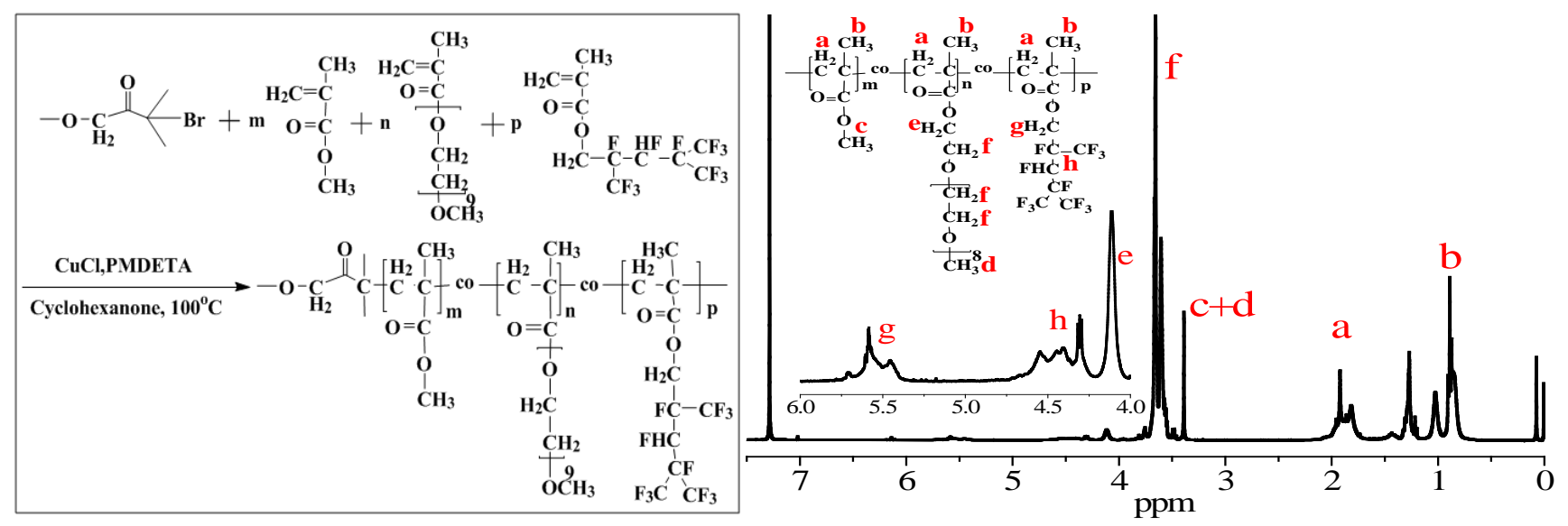

Fig. 1 Synthetic scheme and 1H-NMR spectra of P(PEGMA)-co-P(DFHM)-co-P(MMA)

Table 1 The recipes and detailed polymerization conditions for prepared samples

\begin{tabular}{lllllllllll}
\hline Sample & $\begin{array}{l}\text { MMA/ } \\
\text { PEGMA/ } \\
\text { DFHM }\end{array}$ & $\begin{array}{l}\text { EBiB } \\
\text { /g }\end{array}$ & $\begin{array}{l}\text { CuCl } \\
\text { /g }\end{array}$ & $\begin{array}{l}\text { MMA } \\
\text { /g }\end{array}$ & $\begin{array}{l}\text { PEGMA } \\
\text { /g }\end{array}$ & $\begin{array}{l}\text { DFH } \\
\text { /g }\end{array}$ & $\begin{array}{l}\text { PMDETA } \\
\text { /g }\end{array}$ & $\begin{array}{l}\text { Cyclohex- } \\
\text { anone } \\
/ m l\end{array}$ & $\begin{array}{l}\text { Mp/ } \\
\times 10^{4}\end{array}$ & PDI \\
\hline $\begin{array}{l}\text { Sample } \\
1\end{array}$ & $14 / 1 / 1.2$ & 0.02 & 0.01 & 14.0 & 0.50 & 2.40 & 0.035 & 10 & $\begin{array}{l}2901 \\
9\end{array}$ & 1.47 \\
$\begin{array}{l}\text { Sample } \\
2\end{array}$ & $20 / 1 / 0.8$ & 0.02 & 0.01 & 10.0 & 0.25 & 2.23 & 0.035 & 10 & 2302 & 1.32 \\
$\begin{array}{l}\text { Sample } \\
3\end{array}$ & $14 / 1 / 0.4$ & 0.02 & 0.01 & 14.0 & 0.50 & 1.20 & 0.035 & 10 & $\begin{array}{l}2102 \\
0\end{array}$ & 1.11 \\
\hline
\end{tabular}

Characterization. Proton nuclear magnetic resonance $\left({ }^{1} \mathrm{H}-\mathrm{NMR}\right)$ and gel permeation chromatography (GPC, Waters1515) were used for the chemical structural of P(MMA)-co-P(PEGMA)-co-P(DFHM). The self-assembly behavior of $\mathrm{P}(\mathrm{MMA})$-co-P(PEGMA)-Co-P(DFHM) in $\mathrm{CHCl}_{3}$ solution $\left(0.01 \mathrm{~g} \cdot \mathrm{ml}^{-1}\right)$ was observed by transmission electron microscopy (TEM, JEM-3010). The films (prepared by casting $5 \mathrm{wt} \%$ homogeneous solutions in $\mathrm{CHCl}_{3}$ onto glass slides and dried at ambient temperature for $72 \mathrm{~h}$ ) were characterized for the static contact angle (SCA) on T200-Auto (OneAttension), the surface topographies and roughness (root-mean-square roughness) by NT-MDT new Solver-Next atomic force microscopy (AFM), and the surface composition by X-Ray photoelectron spectroscopy (XPS). The thermal stability of copolymer was evaluated by thermo gravimetric analysis (TGA,STA449C Jupiter from NETZSCH). The adsorption of water and bovine serum albumin (BSA) on the surfaces of films was monitored using a Q-Sense E4 (Sweden) quartz crystal microbalance with dissipation monitoring (QCM-D).

\section{Results and Discussion}

The self-assembly of P(PEGMA)-co-P(DFHM) in solutions. Self-assembled colloids of $\mathrm{P}(\mathrm{MMA})$-co-P(PEGMA)-co-P(DFHM) is depended on the proportion of hydrophilic and hydrophobic segment in three copolymers (Table 1), as shown in TEM results (Fig. 2). Because the solubility of PDFHM segments is much lower than $\mathrm{P}(\mathrm{PEGMA})$ and PMMA in $\mathrm{CHCl}_{3}$ solution, the micelle size increases with the reduction of PDFHM content, which makes three samples are mainly presented dichroic hemispere micelles in the size of 150-200nm (Fig. 2a), $200-250 \mathrm{~nm}$ (Fig. 2b) and 250-300nm (Fig.2c). The micelles are composed of light P(PEGMA)/PMMA chains and dark P(DFHM) chains because of good solubility of P(PEGMA) and P(MMA) segments that easily stretches to form a loose and big particle, while the dark park of $\mathrm{P}(\mathrm{DFHM})$ chains relatively get together tightly. These 
reflect that the micelles structures are consistent with the copolymer composition. On the other hand, DSL analysis shows that the vast majority of micelle particles (about 50\%) are 164-192 nm, 220-255 $\mathrm{nm}$ and 255-300 $\mathrm{nm}$ in $\mathrm{CHCl}_{3}$ solution, which is well agreed with the TEM result. But the diameter distribution of particles is a little wide, which is related to the disordered structure of P(MMA)-co-P(PEGMA)-co-P(DFHM) copolymer.

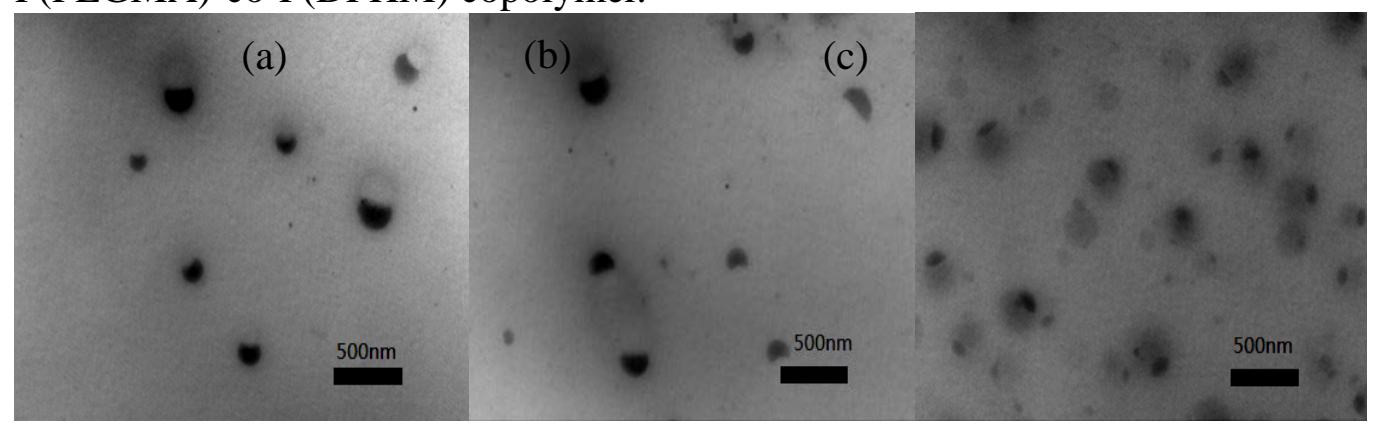

Fig.2 TEM of P(PEGMA)-co-P(DFHM)-co-P(MMA) in CHCl3 solution (a-c: Sample 1-3)

The properties of polymer films. The surface roughness is increased with the decreasing of fluorine content in P(MMA)-co-P(PEGMA)-co-P(DFHM), Sample 1 (MMA/PEGMA/DFHM=14/1/1.2) with the highest fluorine content has a minimum Ra $(0.343 \mathrm{~nm}$, Fig.3a), Sample 2 (MMA/PEGMA/DFHM= 14/1/0.4) with the lowest fluorine content gives the largest film surface roughness $(\mathrm{Ra}=1.01 \mathrm{~nm}$, Fig.3c), Sample 2 (MMA/PEGMA/DFHM=20/1/0.8) shows Ra $=0.754 \mathrm{~nm}$, Fig.3b). The columnar and island structure are formed in Sample 2 (Fig.3b) and Sample 3 (Fig.3c). Therefore, the larger particles (Fig.2) tends to have higher roughness of film surface, because the larger sphere particles are easy to form gaps between themselves after the evaporation of solvent and produced the greater roughness.

Furthermore, the surface chemical composition of P(MMA)-co-P(PEGMA)-co-P(DFHM) films is explained by XPS in Fig.3d-f. The fluorine content changes from $8.42 \mathrm{wt} \%$ to $17.12 \mathrm{wt} \%$, which is proportional to the content of the fluorine segment and is higher than the theoretical content of solid polymer (8.22\%, 6.47\% and 4.10\% from Fig.3d to Fig.3f). Therefore, the fluorine-contained chains are migrated onto the film surface. Combining with the self-assembled colloids, it can be speculated that this may be associated with the hemisphere figuration of the copolymer particles in $\mathrm{CHCl}_{3}, \mathrm{P}(\mathrm{PEGMA})$ and PMMA segments are likely to produce much interaction to make the film with much carriers because of hydrogen bonding, but the other side which contain a lot of fluorine segments will exposed to the outside. These results are also coincided with the static contact angle and surface free energy.

The static contact angle (SCA) and the surface free energy of all films as shown in Table 2. The water contact angle is increased from $68.52^{\circ}$ to $122.74^{\circ}$ (Fig.4a-c) for three samples from hydrophilic to hydrophobic with the increasing of fluorine content. But the hexadecane contact angles are relatively high $\left(54.73^{\circ}, 55.34^{\circ}\right.$ and $55.41^{\circ}$, Fig4d-f) with the slight changes for three samples to show remarkable oleophobic property. All the results prove that a small amount of fluorine content will perform enough resistance to oil, while P(PEGMA) only increase significantly the hydrophilicity of the surface without affecting the oleophobic property. Therefore, the copolymer containing the highest fluorine content shows the lowest surface free energy $\left(17.31 \mathrm{mN} \cdot \mathrm{m}^{-1}\right)$, as shown in Table 2 .

Table 2 Static contact angles (SCA) and surface free energies of P(PEGMA) -co-P(DFHM) -co-P(MMA)

\begin{tabular}{lllllc}
\hline Samples & $\theta($ water $) /^{\circ}$ & $\theta$ (hexadecane) $/^{\circ}$ & $\gamma / \mathrm{mN} \mathrm{m}^{-1}$ & $\Delta \mathrm{f} / \mathrm{Hz}$ & $\Delta \mathrm{D} / \times 10^{-6}$ \\
\hline Sample 1 & $122.74^{\circ}$ & $54.73^{\circ}$ & 17.31 & -120 & 77 \\
Sample 2 & $97.86^{\circ}$ & $55.34^{\circ}$ & 20.03 & -212 & 91 \\
Sample 3 & $68.52^{\circ}$ & $55.41^{\circ}$ & 35.2 & -275 & 110 \\
\hline
\end{tabular}




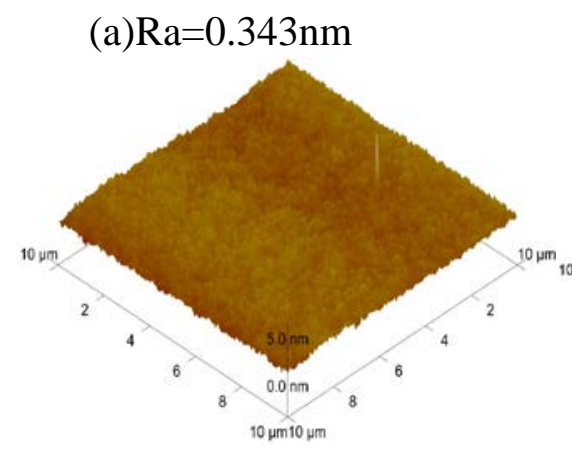

(d)

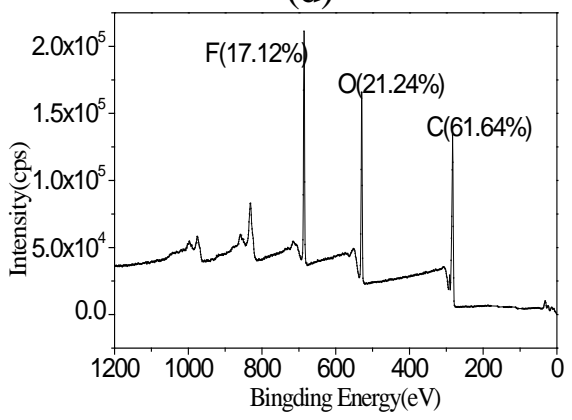

(b) $\mathrm{Ra}=0.754 \mathrm{~nm}$

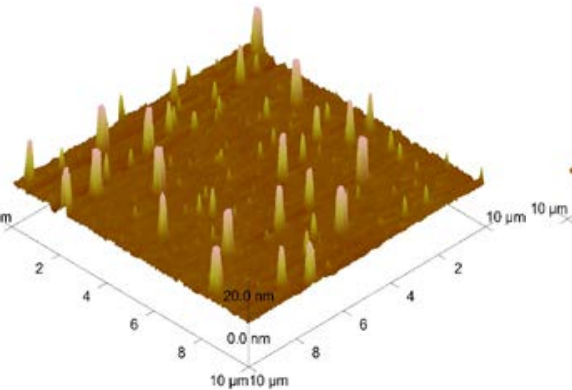

(e)

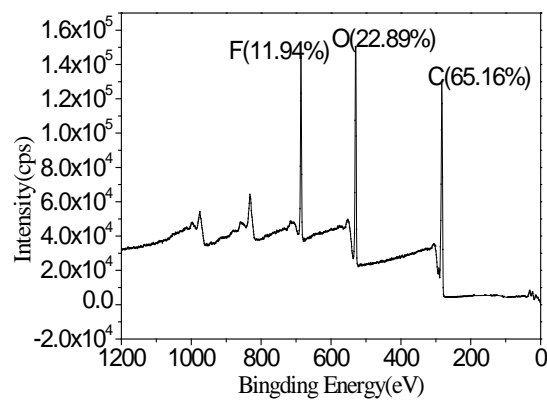

(c) $\mathrm{Ra}=1.01 \mathrm{~nm}$

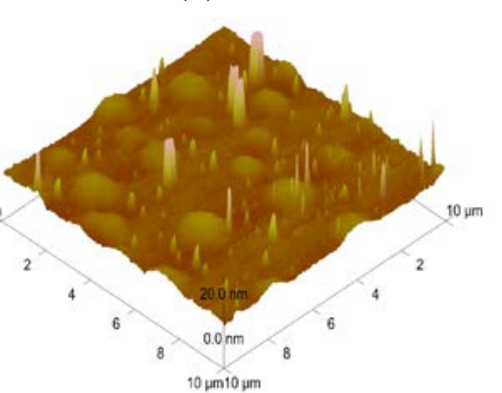

(f)

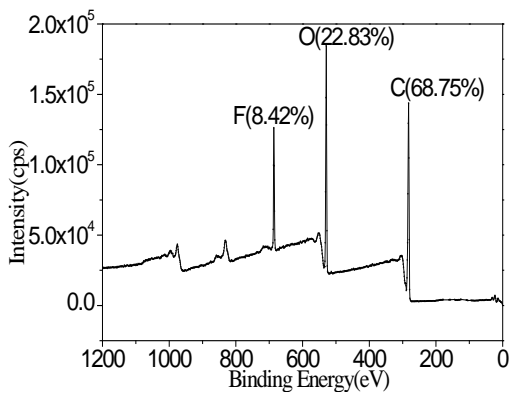

Fig.3 AFM (a-c: Sample 1-3) and XPS result of P(PEGMA)-co-P(DFHM) -co-P(MMA) surfaces (d-f: Sample 1-3)

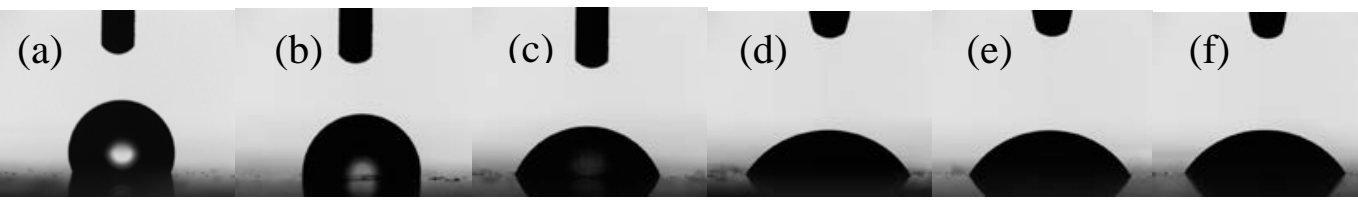

Fig.4 The photos of the content angles of film surface (a-c: water content angles of Sample 1-3; d-f: hexadecane contact angles of Sample 1-3)

Protein resistance of films. QCM-D monitor is used to investigate the adsorption of BSA in Fig.6 according to the changes of resonance frequency $(\Delta \mathrm{f})$ and dissipation $(\Delta \mathrm{D})$. The films are exposed to a solvent of BSA $\left(0.5 \mathrm{mg} \cdot \mathrm{ml}^{-1}\right)$ with the base line of PBS $(\mathrm{pH}=7.4)$. As shown in Fig.6a, $\Delta \mathrm{f}$ decreases quickly when introducing BSA at about 60 minutes, which indicates that the film surface starts to adsorb BSA. At the same time, the related increase of $\Delta \mathrm{D}$ in Fig.6b illustrates the adsorbed protein layer is not viscous $\left(\Delta \mathrm{D}=3.0-5.2 \times 10^{-6}\right)$. At 90 minutes, a subsequent rinsing of PBS produces almost no change which suggesting a less amount of adsorbed BSA is dissociated when rinsing with PBS, and the majority of the protein adsorbed irreversibly on the films. In comparison with the water adsorption $(\Delta \mathrm{f}=-128 \sim-175 \mathrm{~Hz})$, the surface adsorb lesser BSA $(\Delta \mathrm{f}=-15 \sim-25 \mathrm{~Hz})[14]$. As is shown in Fig.6a, the film surface with much PEGMA segments adsorb less BSA protein [15]. The reliable explanation is that the long side chains of P(PEGMA) produce steric effect and that the methoxyl groups in P(PEGMA) are combined with a large number of water molecules on the film surface via hydrogen bonding, so that there is a protection layer on the film surface to prevent protein from adsorbing. 

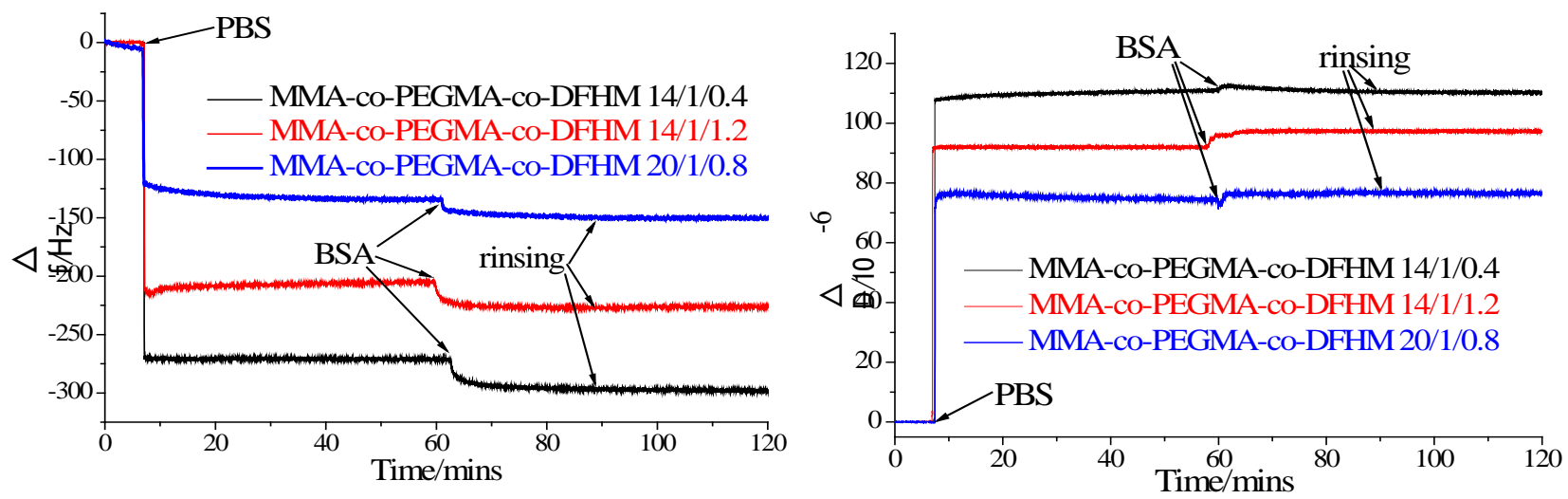

Fig.6 QCM-D real-time change in $\Delta \mathrm{f}$ (a) and $\Delta \mathrm{D}$ (b) of film surfaces

\section{conclusions}

1. Synthesis: The amphiphilic fluoroacrylate copolymers of P(MMA)-co-P(PEGMA)-co-P(DFHM) are synthesized via ATRP.

2.Self-assembly: The copolymer could spontaneously self-assemble into semisphere-structured micelles in $\mathrm{CHCl}_{3}$ solution with a diameter of $180 \mathrm{~nm}-280 \mathrm{~nm}$. These self-assembled micelles contribute to the rising of surface roughness $(\mathrm{Ra}=0.343-1.010 \mathrm{~nm})$ of films with the decrease of fluorine content.

3. Film properties: The water contact angle $\left(68.52^{\circ}-122.74^{\circ}\right)$ is increased but hexadecane contact angles $\left(54.73^{\circ}-55.41^{\circ}\right)$ is invariant with the increasing of fluorine content, and therefore the surface free energies are decreased $(35.2-17.31 \mathrm{mN} / \mathrm{m})$ because of the migrating of fluorine-containing chains. $\mathrm{P}(\mathrm{MMA})$-co-P(PEGMA)-co-P(DFHM) gives improved $\mathrm{Td}\left(248.9^{\circ} \mathrm{C}\right)$ and better BSA protein-resistance behavior with the increase of $\mathrm{P}(\mathrm{PEGMA}) \operatorname{segment}(\Delta \mathrm{f}=-15 \sim-25 \mathrm{~Hz})$. Therefore, $\mathrm{P}(\mathrm{MMA})$-co-P(PEGMA)-co-P(DFHM) is promised a coating material with low surface free energy and protein absorption.

\section{Acknowledgement}

This work was funded by the National Basic Research Program of China (973 Program, No.2012CB720904), the National Natural Science Foundation of China (NSFC Grants 51373133, 51573145), the International Cooperation Project of Shaanxi Province (No.2014KW11) and the State Administration of Cultural Heritage (20110128).

\section{References}

[1] F.L. Hatton, P. Chambon, T.O. McDonald, A. Owen, S.P. Rannard, Hyperbranched polydendrons: a new controlled macromolecular architecture with self-assembly in water and organic solvents, Chem. Sci. 5(2014)1844-8153.

[2] J.L Liu, W.W He, L.F Zhang, Z.B Zhang, J Zhu, L Yuan, Bifunctional nanoparticles with fluorescence and magnetism via surface-initiated AGET ATRP mediated by an iron catalyst. Langmuir 27 (2011)12684-12692.

[3] F.F Shen, T.H Xiao, L.N Han, S.M Chen, Studies on properties of temperature-sensitive amphiphilic copolymer-modified PVDF ultrafiltration membrane, Desalination and Water Treatment. 54(2015)1507-1518. 
[4] Y.L Shi, S.Y Ma, Synthesis, characterization and release profiles of nanoparticles self-assembled from poly(PEGMA-co-MMA-co-acryloyl- $\beta-C D)$ copolymers, Research article. 47(2015) 270-277.

[5] S.H Lee, M Ouchi, M Sawamoto, Chain Center-Functionalized Amphiphilic Block Polymers: Complementary Hydrogen Bond Self-Assembly in Aqueous Solution, J. Polym. Sci. Part A. 51(2013)4498-4504.

[6] X Chen, G Zhang, Q Zhang, X Zhan, F Chen, Preparation and performance of amphiphilic polyurethane copolymers with capsaicin-mimic and PEG moieties for protein resistance and antibacterial, Ind. Eng. Chem. Fundam. 54 (2015) 3813-3820.

[7] Y.C Chiag, Y Chang, W.Y Chen, R.C Ruaan, Biofouling resistance of ultrafiltration membranes controlled by surface self-assembled coating with PEGylated copolymer, Langmuir. 28 (2012) 1399-1407.

[8] X.L Zhan, G.F Zhang, Q.H Zhang, F.Q Chen, Preparation, surface wetting properties, and protein adsorption resistance of well-defined amphiphilic fluorinated diblock copolymers, Appl. Polym. Sci. 10 (2014) 41167-41178.

[9] E Martinelli, E Guazzelli, C Bartoli, M Gazzarri, F Chiellini, G Galli, M.E Callow, J.A Callow, J.A Finlay, S Hill, Amphiphilic pentablock copolymers and their blends with PDMS for antibiofouling coatings, Polym. Chem. 53 (2015) 1213-1225.

[10] R Prakash, K.V.I.S. Kaler, D.P. Papageorgiou, A.G. Papathanasiou, Performance of multilayered fluoropolymer surface coating for DEP surface microfluidic devices, Microfluid. Nanofluid. 13(2015)309-318.

[11] E.E Fatime, S Deniz, Synthesis of Fluorinated Amphiphilic Block Copolymers Based on PEGMA, HEMA, and MMA via ATRP and CuAAC Click Chemistry, Int. J. Polym. Sci. 10(2014)1155-1165.

[12] X.L Zhan, G.F Zhang, Q.H Zhang, F.Q Chen, Preparation, Surface Wetting Properties, and Protein Adsorption Resistance of Well-Defined Amphiphilic Fluorinated Diblock Copolymers, J. Appl. Polym. Sci. 10(2014)44176-44188.

[13] X Dong, L He, N Wang, J.Y Liang , M.J Niu, X Zhao, Diblock fluoroacrylate copolymers from two initiators: synthesis, self-assembly and surface properties, J. Mater. Chem. 22(2012)23078-23090.

[14] H.P Huang, J Qu, L He, Amphiphilic silica/fluoropolymer nanoparticles: synthesis,tem-responsive and surface properties as protein-resistance coatings, J. Polym. Sci. Part A: Polym.Chem. 54 (2016)381-393.

[15] W.H Guo, J Zhu, Z.P Cheng, Z.B Zhang, X.L Zhu, Anticoagulant surface of 316 L stainless steel modified by surface-initiated atom transfer radical polymerization, Appl. Mater. Interfaces. 3(2011)1675-1680. 